\title{
Studies of coprophilous ascomycetes in Kenya. Podospora species from wildlife dung
}

\section{Mungai $\mathrm{PG}^{1,2,3 *}$, Njogu, $\mathrm{JG}^{3}$, Chukeatirote $\mathrm{E}^{1,2}$ and Hyde $\mathrm{KD}^{1,2}$}

\author{
${ }^{1}$ Institute of Excellence in Fungal Research, School of Science, Mae Fah Luang University, Chiang Rai 57100, Thailand \\ ${ }^{2}$ School of Science, Mae Fah Luang University, Chiang Rai 57100, Thailand \\ ${ }^{3}$ Biodiversity Research and Monitoring Division, Kenya Wildlife Service, P.O. Box 4024100100 Nairobi, Kenya
}

Mungai PG, Chukeatirote E, Njogu JG, Hyde KD 2012 - Studies of coprophilous ascomycetes in Kenya. Podospora species from wildlife dung. Mycosphere 3(6), 978-995, Doi 10.5943 /mycosphere/3/6/12

Moist chamber cultures were made from wildlife dung obtained from national parks in Kenya. Ten dung types produced 28 specimens of Podospora. Five species, Podospora anserina, $P$. argentinensis, $P$. australis, $P$. communis and $P$. minor are described using their morphological features. Podospora minor seems to be a rare species and is recorded for the first time in Kenya. Podospora communis, $P$. anserina and P. australis are the most common species on dung types examined.

Key words - Arnium - biodiversity - conservation - perithecioid - Schizothecium - taxonomy

\section{Article Information}

Received 14 November 2012

Accepted 26 November 2012

Published 29 December 2012

*Coresponding Author: Paul G. Mungai - email - emu@kws.go.ke

\section{Introduction}

A survey of coprophilous fungi in Kenya that we started three years ago has so far produced four publications (Mungai et al. 2012a, 2012b, 2012c, 2012d) on some interesting ascomycetes groups. We have examined Ascobolus Pers., Saccobolus Boud., Schizothecium Corda., and an assortment of less common Sordariales, followed now by this work on Podospora Ces., one of the commonest genera on dung (Lundqvist 1972, Doveri 2004a, 2008, Bell 2005). Podospora is predominantly coprophilous, cosmopolitan and normally perithecioid. This genus is strikingly similar to Schizothecium Corda, Arnium Nitschke ex G. Winter, Zygopleurage Boedijn and Cercophora Fuckel. It has been treated in great detail by Cain (1934), Mirza \& Cain
(1969), Lundqvist (1972), Bell \& Mahoney (1995, 1997) Bell (2005) and Doveri (2004a, 2008).Objectives of this survey are to: i) study the taxonomy of Podospora from Kenyan wildlife dung, ii) document the ecology and biodiversity of Podospora species on different wildlife dung types and, iii) create awareness on the importance of dung fungi in biodiversity conservation and management.

\section{Materials and methods}

Twenty-one samples from ten dung types, African elephant (Loxodonta africana Blum.), black rhinoceros (Diceros bicornis Linn.), Cape buffalo (Syncerus caffer Sparrman), zebra (Equus burchelli Gray), dikdik (Madoqua kirkii Günther), giant forest hog (Hylochoerus meinertzhageni Thomas), 
giraffe (Giraffa camelopardalis Linn.), hippopotamus (Hippopotamus amphibious Linn.), impala (Aepyceros melampus Licht.) and waterbuck (Kobus ellipsiprymnus Ogilbyi) collected from Aberdares Country Club Game Sanctuary, Tsavo East, Aberdares and Nairobi National Parks were cultured in moist chamber. Mungai et al. (2011, 2012a, 2012b) provide details of materials and methods.

Isolation of coprophilous fungi was carried out as previously described by Mungai et al. (2011, 2012a, 2012b). Podospora species identification was then performed using a taxonomic key of Mirza \& Cain (1969), Lundqvist (1972), Doveri (2004a) and Doveri (2008)

\section{Taxonomy}

\section{Podospora Ces.}

Podospora in the subfamily Podosporoideae N. Lundq. (Lasiosphaeriaceae Nannf., Sordariales Chadefaud ex D. Hawksworth and O.E. Eriksson) is characterised by perithecioid, non-stromatic, more or less membranaceous, dark, superficial to semi-immersed ascomata, clavate to saccate, 4- to poly-spored asci, and ascospores 2-celled at maturity with a dark pigmented upper cell, a hyaline lower pedicel and gelatinous equipment usually as caudae. It is closely related to Schizothecium Corda, Arnium Nitschke ex G. Winter, Zygopleurage Boedijn and Cercophora Fuckel. Schizothecium is mainly differentiated from Podospora by having perithecia with swollen agglutinated hairs, particularly gathered at the neck base (Lundqvist 1972, Doveri 2008).

Unlike Podospora, the immature ascospores of Arnium are ellipsoidal or fusiform, while the mature ones are nonpedicellate (Lundqvist 1972, Doveri 2004a, Bell 2005). Zygopleurage differs from Podospora by having ascospores with two opposite dark cells connected by a long cylindrical intercalary cell. Cercophora has hyaline vermiform-sigmoid or cylindricgeniculate ascospores and usually cylindricclaviform asci with a thickened apical ring and often with sub-apical globules (Lundqvist 1969, 1972, Abdullah \& Rattan 1978, Doveri 2008).
Podospora, a cosmopolitan and usually coprophilous genus, was treated in detail by Cain (1934), Mirza \& Cain (1969), Lundqvist (1972), Bell \& Mahoney (1995, 1997), Bell (2005) and Doveri (2004a, 2008). The morphological characters of peridial hairs, peridium, asci and ascospores have been extensively used in generic delimitation (Cain 1934, Mirza \& Cain 1969, Lundqvist 1972, Bell \& Mahoney 1995, 1997, Doveri 2004a, 2008, Chang \& Wang 2005). Significant importance to circumscribe species of Podospora has been attributed to the shape and size of the perithecium and peridial wall cells, the number of ascospores per ascus and the shape of ascospore primary and secondary appendages (Cain 1934, Mirza \& Cain 1969, Lundqvist 1972, Doveri 2004a, 2008, Bell 2005, Chang \& Wang 2005). The presence or absence of hairs or bristles on the perithecia, such as those found on $P$. anserina, $P$. australis and $P$. minor, is another useful tool in species delimitation (Mirza \& Cain 1969, Lundqvist 1972, Doveri 2004a, 2008, Bell 2005). Cultural attributes, though not extensively studied, seem to be important in identifying Podospora spp. or groups of related taxa.

In this study, we have used morphological characters to identify the Podospora species isolated from wildlife dung in Kenya.

Podospora anserina (Ces. ex Rabenh.) Niessel, Hedwigia 22:156, 1883. (Fig. 1A-K)

$=$ Sphaeria anserina Ces., in litt.

$\equiv$ Malinvernia anserina Ces. ex Rabenh., Hedwigia 1: 116, 1856.

= Sphaeria pauciseta Ces. in Rabenh, Kl. Herb. Viv. Myc., ed. 1: 116, 1856.

= Sordaria pauciseta (Ces.) Ces. \& De Not., Comm. Soc. Critt. Ital. 1: 226, 1863.

= Malinvernia pauciseta (Ces.) Fuckel, Fungi Rhen. 1002, 1864.

= Malinvernia breviseta Fuckel, Jahrb.

Nass. Ver. Naturk. 24: 243, 1870.

= Sordaria anserina (Ces.ex Rabenh.) G.

Winter, Bot. Zeit. 31: 483, 1873.

= Sphaeria breviseta (Fuckel) W. Philips \&

Plowr., Grevillea 2: 187, 1874.

= Sordaria anserina f. ovina Sacc., Mycoth. Ven. 1179, 1878. 

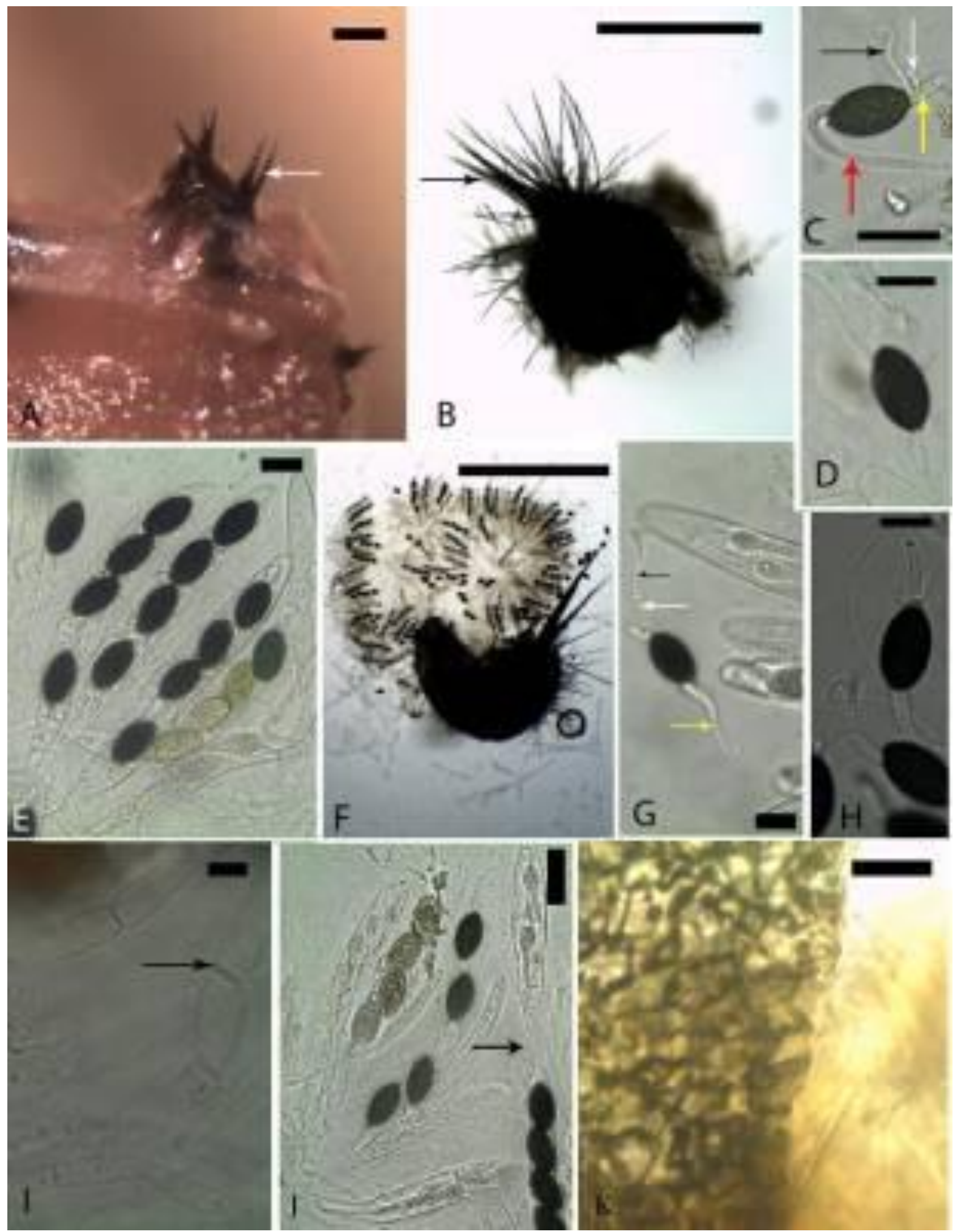

Fig. 1 - Podospora anserina (KWSANP004-2009). A. Ascomata on dung, note tufts of pointed hairs (arrows). B Whole ascoma water mount, note hairs (arrows). C-D, G-H Mature ascospores showing pedicels and caudae (arrows). E Asci and ascospores showing uniseriate spore arrangement. F Squashed ascoma. I Paraphyses, note septation (arrow). J Ascus showing stipe (arrow). K Details of exoperidium. Scale bars: $\mathbf{A}-\mathbf{B}=200 \mu \mathrm{m}, \mathbf{C}-\mathbf{D}=20 \mu \mathrm{m}, \mathbf{E}=50 \mu \mathrm{m}, \mathbf{F}=500$ $\mu \mathrm{m}, \mathbf{G}=50 \mu \mathrm{m}, \mathbf{H}-\mathbf{K}=20 \mu \mathrm{m}$. 
= Hypocopra erecta Speg., Anal. Soc. Cient. Argent. 10: 15, 1880.

= Sordaria erecta (Speg.) Sacc. Syll. Fung. 1: $239,1882$.

= Podospora erecta (Speg.) Niessl, Hedwigia 22: 156, 1883.

= Sordaria pencillata Ellis \& Everh., Journ. Mycol. 4: 78, 1888.

$=$ Podospora pencillata (Ellis \& Everh.) Ellis \& Everh., North Amer. Pyren.: 131, 1892.

$=$ Pleurage anserina (Ces. ex Rabenh.) Kuntze, Rev. Gen. Plant. 3(3): 504, 1898.

$=$ Pleurage erecta (Speg.) Kuntze, Rev. Gen. Plant. 3(3): 505, 1898.

= Pleurage pencillata (Ellis \& Everh.) Kuntze, Rev. Gen. Plant. 3 (3):505, 1898.

= Sordaria communis (Speg.) Sacc. va. tetraspora Speg., Annal. Mus. Nac. Buenos Aires 6: 253, 1899.

= Podospora pauciseta (Ces.) Traverso, Fl. Ital. Crypt. 1, Fungi 1:431, 1907.

$\equiv$ Bombardia anserina (Ces. ex Rabenh.) Mig., Thomes Krypt. Fl. 10 (1): 123, 1912. $\equiv$ Schizothecium anserinum (Ces. ex Rabenh.) E.A. Bessey, Morph. Tax. Fungi:264, 1950.

= Podospora filiformis Cailleux, Cah. Maboke 7:102, 1969.

(Adopted from Doveri 2008)

Ascomata perithecioid, semi-immersed to nearly superficial, 380-600 $\mu \mathrm{m}$ high, 300$500 \mu \mathrm{m}$ diam., scattered or in small groups, covered with few black tubercles and numerous hyphoid hairs, conical or pyriform; neck subcylindric, $90-120 \mu \mathrm{m} \times 70-80 \mu \mathrm{m}$, blackish, coriaceous, with few tufts of agglutinated, rigid, straight, brown, pointed hairs, 100-300 $\mu \mathrm{m}$ long, continuous or sparingly septate. Peridium pseudoparenchymatous: endostratum of pale translucent thin-walled polygonal cells 10-25 $\mu \mathrm{m}$ wide; exostratum of thick-walled polygonal cells where hyaline wavy septate hairs originate, 2-4 $\mu \mathrm{m}$ wide. Paraphyses cylindricmoniliform, exceeding the asci, 4.5-6.5 $\mu \mathrm{m}$ broad, septate, with hyaline vacuoles. Asci 4spored, 175-275 × 22-29 $\mu \mathrm{m}$, clavate, with a thin apical ring, long and slender, lobate stipe. Ascospores obliquely uniseriate, two-celled at maturity: spore head $32-36 \times 17-20 \mu \mathrm{m}$, immature spoon shaped, mature ellipsoidal, somewhat unequilateral, smooth, dark brown, with a centric germ pore; lower cell (pedicel) cylindrical, 20-30 × 4-5 $\mu \mathrm{m}$, slightly pointed; upper cauda, sub-apical, fairly long, lash-like, furrowed, tip curved, 30-80 × 6.5-8 $\mu \mathrm{m}$ at base; lower cauda solid, filiform; additional two to three, short, filiform caudae, with hooked tips, attached to basal part of primary appendage near septum.

Material examined -7 isolates from dung incubated for between 6 and 20 days KENYA, Aberdare Country Club Game Sanctuary, Central Province, bushed grassland, GPS S00 19 28.1 E036 55'54.3”, altitude 2161 $\mathrm{m}$, giraffe, 29 August 2009, P. Mungai, KWSACC003-2009; Aberdare National Park, Central Province, montane forest, GPS, S00²1'42.0" E036'52'55.9', altitude $2076 \mathrm{~m}$, black rhinoceros, 29 August 2009, KWSANP004-2009; Nairobi National Park, Nairobi Province, bushed grassland, GPS S01 ${ }^{\circ} 20^{\prime} 50.1^{\prime \prime}$ E036 47'51.3", altitude $1695 \mathrm{~m}$, hippopotamus, 31 August 2009, P. Mungai, KWSNNP012-2009; Nairobi National Park, Nairobi Province, bushed grassland, GPS 0252737 9847748, altitude $1876 \mathrm{~m}$, Cape buffalo, 27 August 2009, P. Mungai, KWSNNP004-2009; Tsavo East National Park, Coast Province, riverine habitat, GPS S0321'064" E038 37'501', altitude $514 \mathrm{~m}$, Cape buffalo, 23 September 2008, P. Mungai, KWSTE007A-2008; Tsavo East National Park, Coast Province, riverine, GPS S0302'29.7”, E038 41'35.8", altitude $354 \mathrm{~m}$, dikdik, 27 August 2009, P. Mungai, KWSTE005B-2009; Tsavo East National Park, Coast Province, riverine, GPS S0321'666', E038 38'772', altitude $514 \mathrm{~m}$, African elephant, 23 August 2009, P. Mungai, KWSTE005A-2009.

Notes - Pododpora anserina sect. Malinvernia Rabenh. is distinguished from $P$. australis (Speg.) Niessl sect. Andreanszkya Tóth. by the former's smaller non-apiculate ascospores with more complex furrowed caudae and the latter's notably simpler taenioid caudae (Lundqvist 1972, Doveri 2004a, 2008, Bell 2005). The morphology of these collections agree with that observed for this taxon in previous examinations (Cain 1934, Lundqvist 1972, Bell 1983, 2005, Doveri 2004a, 2008). This is a common species on wildlife dung in Kenya. 

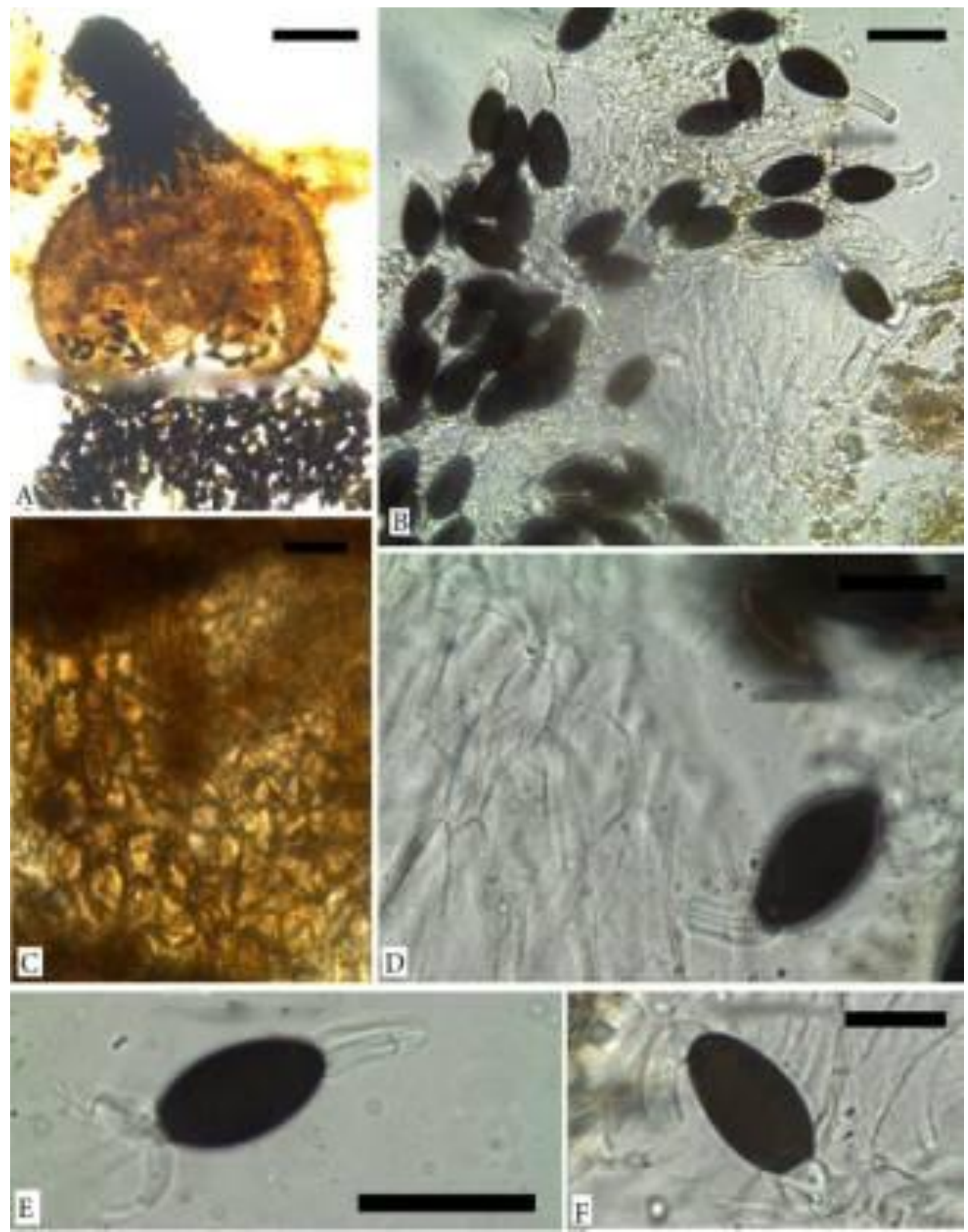

Fig. 2 - Podospora argentinensis (KWSACC002-2009). A Ascoma squash. B Free mature ascospores. C Details of exoperidium. D Paraphyses. E-F Free mature ascospores showing pedicel and caudae. Scale bars: $\mathbf{A}=200 \mu \mathrm{m}, \mathbf{B}=50 \mu \mathrm{m}, \mathbf{C}-\mathbf{F}=20 \mu \mathrm{m}$. 
Podospora argentinensis (Speg.) J.H. Mirza \& Cain, Can. J. Bot. 47: 2008, 1969. (Fig. 2A-F) $\equiv$ Sordaria argentinensis Speg., Anal. Mus. Nac. Buenos Aires 23:49, 1912. $\equiv$ Pleurage argentinensis (Speg.), C. Moreau, Encycl. Mycol. 25: 252, 1953.

Ascomata perithecioid, immersed or semi-immersed, 400-670 $\mu \mathrm{m}$ high, 200-500 $\mu \mathrm{m}$ diam., scattered, upper portion of perithecia with black tubercles, globose to pyriform. Peridium membranaceous; endoperidium of thin-walled polygonal cells; exoperidium composed of brownish thin translucent textura globulosa-angularis, cells measuring 6-11 $\times$ 5-7 $\mu \mathrm{m}$. Neck short cylindrical, fully covered with black papillae. Paraphyses numerous, filiform above, ventricose below, fugacious. Asci 8-spored, 180-200 × 30-40 $\mu \mathrm{m}$, clavate, narrowly rounded at apices, long stipitate. Ascospores biseriate, two-celled: spore head 27.5-35 $\times$ 15-19 $\mu \mathrm{m}$, ovoid-ellipsoidal, smooth, brown to blackish, thin-walled, with an eccentric germ pore; pedicel often appearing twisted, flattened, cylindrical, or slightly clavate 18-23 $\mu \mathrm{m}$ long, $6.5-9 \mu \mathrm{m}$ broad at base; upper caudae formed as a lyre-shaped structure, sometimes elongated; lower caudae, fugacious, small, formed as a whorl at the proximal end of the pedicel.

Material examined - 2 isolates from dung incubated 34 and 43 days - KENYA, Nairobi National Park, Nairobi Province, GPS 37M0257082, 9850692, altitude $1668 \mathrm{~m}$, zebra, 20 August 2010, P. Mungai, KWSNNP0162010; Aberdare Country Club Game Sanctuary in Central Province, S00 19'28.1" E036 55'54.3", altitude $2161 \mathrm{~m}$, zebra, bushed grassland, 30 August 2009, P. Mungai, KWSACC002-2009.

Notes - Podospora argentinensis sect. Rhypophila Lundq. is closely related to $P$. decipiens (G. Winter ex Fuckel) Niessl of the same section but the former has smaller ascospores (Mirza \& Cain 1969, Doveri 2004a, Bell 2005) while the latter does not have a cauda attached at the end of the pedicel (Chang \& Wang 2004). This species has been recorded many times from tropical areas and is deemed to be this region's substitute of $P$. decipiens (Mirza \& Cain 1969, Lundqvist 1973, Krug \& Khan 1989, Richardson 2001), which is common in temperate areas. The characters of the Kenyan collection are similar to the descriptions made in previous studies (Mirza \& Cain 1969, Bell 2005).

Podospora australis (Speg.) Niessl, Hedwigia 22: 156, 1883. (Figs. 3A-G, 4A-G)

$\equiv$ Hypocopra australis Speg. Anal. Soc. Cient. Argent. 10: 137, 1880.

$\equiv$ Sordaria australis (Speg.) Sacc., Syll. Fung. 1:239, 1882.

$\equiv$ Pleurage australis (Speg.) Kuntze, Rev. Gen. Plant. 3(3): 505, 1898.

$=$ Sordaria apiculifera Speg. Anal. Mus. Nac. Buenos Aires 6: 251, 1899.

$=$ Pleurage taenioides Griffiths, Mem. Torrey Bot. Club 11: 58, 1901.

$=$ Sordaria taenioides (Griffiths) Sacc., Syll. Fung. 17: 602, 1905.

= Sordaria macrura A. Bayer, Acta Soc. Nat. Moraviae 1: 95, 1924.

= Pleurage apiculifera (Speg.) C. Moreau, Encycl. Mycol. 25: 252, 1953.

$=$ Podospora taenioides (Griffiths) Cain, Can. J. Bot. 40: 460, 1962.

= Podospora apiculifera (Speg.) Mirza \& Cain, Can. J. Bot. 47: 2006, 1969.

(Adopted from Doveri 2004a)

Ascomata perithecioid semi-immersed to superficial, $550-850 \mu \mathrm{m}$ high to $500-620$ $\mu \mathrm{m}$ diam., scattered, olive-brown to nearly black, pyriform; the exposed portion covered with long, septate, branched, olivaceous brown and hyaline tipped hairs $2.5-3 \mu \mathrm{m}$ broad. Peridium semi-membranaceous, translucent to slightly opaque; endoperidium of textura angularis cells; exoperidium of thick-walled textura angularis cells $8-11 \times 6.5-8.5 \mu \mathrm{m}$. Neck 180-240 × 180-190 $\mu$ m, black, opaque, papilliform to cylindrical; with, septate, rigid hairs 27.5-38.5 $\mu \mathrm{m}$ long, 2-4 $\mu \mathrm{m}$ broad at base. Paraphyses cylindric-moniliform or filiform, longer than asci, 5-6 $\mu \mathrm{m}$ broad, septate, rarely branched. Asci 4-spored, 258-307 × 36.5-44 $\mu \mathrm{m}$, unitunicate, narrowly cylindrical, tapering below into a slender very long stipe, with an indistinct apical apparatus, apex narrow. Ascospores obliquely uniseriate at maturity, two-celled: spore head 50.5-55.5 × 25-27 $\mu \mathrm{m}$, narrowly ovoid to ellipsoidal, symmetrical, dark brown, smooth, thick walled, flattened at base with an apical conspicuous germ pore; lower cell (pedicel) reduced to a conspicuous 
Mycosphere Doi 10.5943/mycosphere/3/6/12 


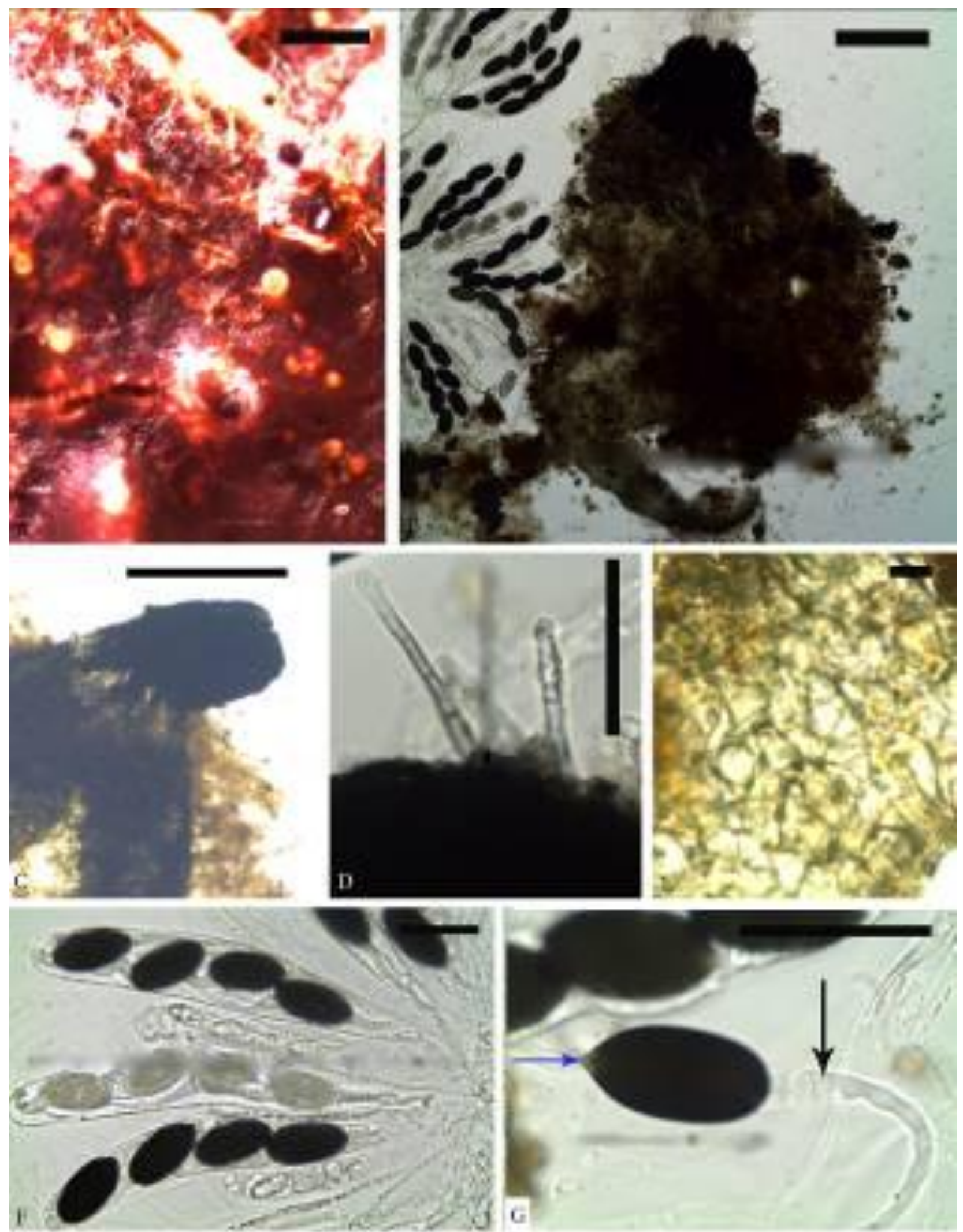

Fig. 3 - Podospora australis (KWSACC002-2009). A Necks of semi-immersed ascomata on dung. B Ascoma squash. C Perithecial neck. D Septate and hyaline perithecial hairs. E Details of exoperidium. F Mature and immature asci. G Free mature ascospore showing taenioid caudae (black arrow) and apiculum (blue arrow). Scale bars: $\mathbf{A}=1000 \mu \mathrm{m}, \mathbf{B}-\mathbf{C}=200 \mu \mathrm{m}, \mathbf{D}-\mathbf{E}, \mathbf{G}=20$ $\mu \mathrm{m}, \mathbf{F}=50 \mu \mathrm{m}$. 

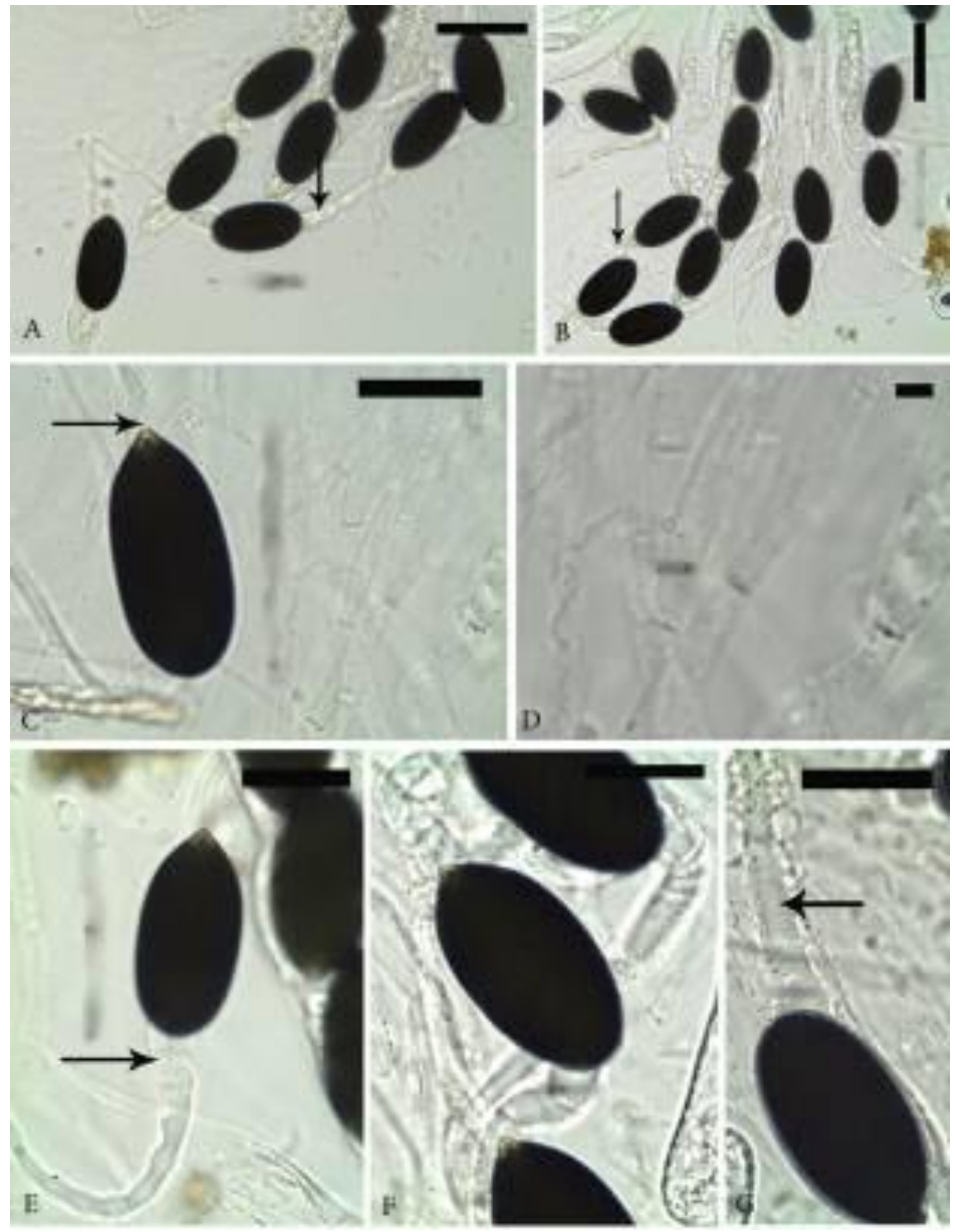

Fig. 4 - Podosdpora australis (KWSACC002-2009). A-B Free mature ascospores, note gelatinous equipment (arrows). C Mature ascospore among paraphyses, note apiculum (arrow). D Details of paraphyses. E-G Details of mature ascospores, note caudae with transverse banding (arrows). Scale bars: $\mathbf{A}-\mathbf{B}=50 \mu \mathrm{m}, \mathbf{C}-\mathbf{G}=20 \mu \mathrm{m}$. 
small basal triangular apiculum, hyaline, 4-6.5 $\times 2-3 \mu \mathrm{m}$; upper cauda 50-100 $\mu \mathrm{m}$ long, 8-15 $\mu \mathrm{m}$ broad at the base, more segmented (taenioid) in proximal part, longitudinally furrowed and poly-channeled, wavy, attached slightly eccentrically, not covering the germ pore; lower cauda similar, long, originating from the basal end of spore, completely covering the apiculum, hollow and taenioid.

Material examined - 4 isolates from dung incubated between 12 and 21 days KENYA, Aberdare Country Club Game Sanctuary, Central Province, GPS S00 19'28.1' E036 55'54.3”, altitude $2161 \mathrm{~m}$, zebra, 30 August 2009, P. Mungai, KWSACC002-2009; Nairobi National Park, Nairobi Province, GPS 37M0257532, 9848948, altitude $1647 \mathrm{~m}$, giraffe, 20 August 2010, P. Mungai, KWSNNP017A-2010; Nairobi National Park, Nairobi Province, GPS 37M0257532, 9848948, altitude $1647 \mathrm{~m}$, giraffe, 20 August 2010, P. Mungai, KWSNNP017B-2010; Nairobi National Park, Nairobi Province, GPS 37M0255297, 9848528, altitude $1693 \mathrm{~m}$, zebra, 20 August 2010, P. Mungai, KWSNNP0162010.

Notes - Podospora australis sect. Andreanszkya (Tóth) Lundq., noted for having a reduced pedicel, is quite unique and is identified by its large ascospores with a small, basal apiculum and taenioid caudae, one on each side of the spore head (Mirza \& Cain 1969, Lundqvist 1972, Doveri 2004a, 2008, Bell 2005). It is differentiated from the related $P$. anserina by its larger apiculate ascopores and longitudinally poly-channeled and transversely banded caudae (Lundqvist 1972, Doveri 2004a, Bell 2005). Lundqvist (1972) argues that $P$. apiculifera (Speg) Mirza and Cain is a depauperate form of Podospora australis. Previous records for this taxon in Kenya are from the dung of steenbok, impala, bushbuck, hippopotamus, buffalo, zebra, eland zebra, giraffe and cattle (Caretta et al. 1998, Krug \& Khan 1989). It seems to be a very common species on wildlife dung.

Podospora communis (Speg.) Niessl, Hedwigia 22: 156, 1883. (Figs. 5A-E, 6A-E)

$\equiv$ Hypocopra communis Speg., Anal. Soc.

Cie. Argent. 10: 14, 1880.

$\equiv$ Sordaria communis (Speg.) Sacc. Syll.
Fung. 1: 231, 1882.

$=\quad$ Sordaria vestita Zopf, Zeits. Naturw. 56: 556, 1883.

= Podospora vestita (Zopf) G. Winter, Rabenh. Krypt. Fl. 1 (2): 176, 1885.

$=$ Sordaria macrostoma Speg., Anal. Mus. Nac. Buenso Aires 6: 252, 1899.

$=$ Pleurage vestita (Zopf) Griffiths, Mem. Torrey Bot. Club 11: 76, 1901.

= Bombardia vestita (Zopf). Mig., Thome's Krypt. Fl. 10 (1): 126, 1913.

$=$ Sordaria occidentalis Bat. \& Pontual, Bol. Agric. Pernambuco 15: 38, 1948.

= Pleurage macrostoma (Speg.) C. Moreau, Encyl. Mycol. 25: 262, 1953.

(Adopted from Doveri 2004a)

Ascomata perithecioid, superficial, 670-1100 × 410-530 $\mu \mathrm{m}$, scattered or gregarious in small groups, semi-transparent, becoming olivaceous or dark brown, adorned with fine brown hairs of over $40 \mu \mathrm{m}$ long, disappearing with age, obpyriform. Neck conical or cylindrical, 150-385 × 130-150 $\mu \mathrm{m}$, roughened with small, black papillae or glabrous, opaque, usually curved; ostiole over $80 \mu \mathrm{m}$ diam. Peridium membranaceous, semitransparent; endoperidium of textura angularis cells; exoperidium consisting of yellowisholive textura angularis cells. Paraphyses reduced to a mass of elongated vesicles. Asci 8spored, 240-265 × 40-50 $\mu \mathrm{m}$, unitunicate, clavate, with a narrow tapering broad apex, fairly long stipe, apical ring thin and barely visible. Ascospores biseriate, two-celled: spore head 29-40 × 16-25 $\mu \mathrm{m}$, at first sub-cylindric, later ellipsoidal with truncate base, occasionally asymmetrical, dark to olivaceous brown, opaque, thick walled, with a minute apical germ pore; pedicel 25-42 × 5-6 $\mu \mathrm{m}$, cylindrical, wider at the base, hyaline, slightly curved in the early stages, straight later; upper caudae four, flattened, short, independent, curved, composed of two filaments, lash-like, larger, $40-50 \times 2-4 \mu \mathrm{m}$ diam, sub-apical, surrounding the germ pore; lower caudae four, 3.5-5 $\times$ 1.5-2.5 $\mu \mathrm{m}$, curved, each independently arising from apex of the pedicel, lash-like, $3.5-5 \times 1.5-2.5 \mu \mathrm{m}$.

Material examined - 14 isolates from dung incubated between 12 and 64 days KENYA, Nairobi National Park, Nairobi Province, UTM37 02537515 M 9849130, 


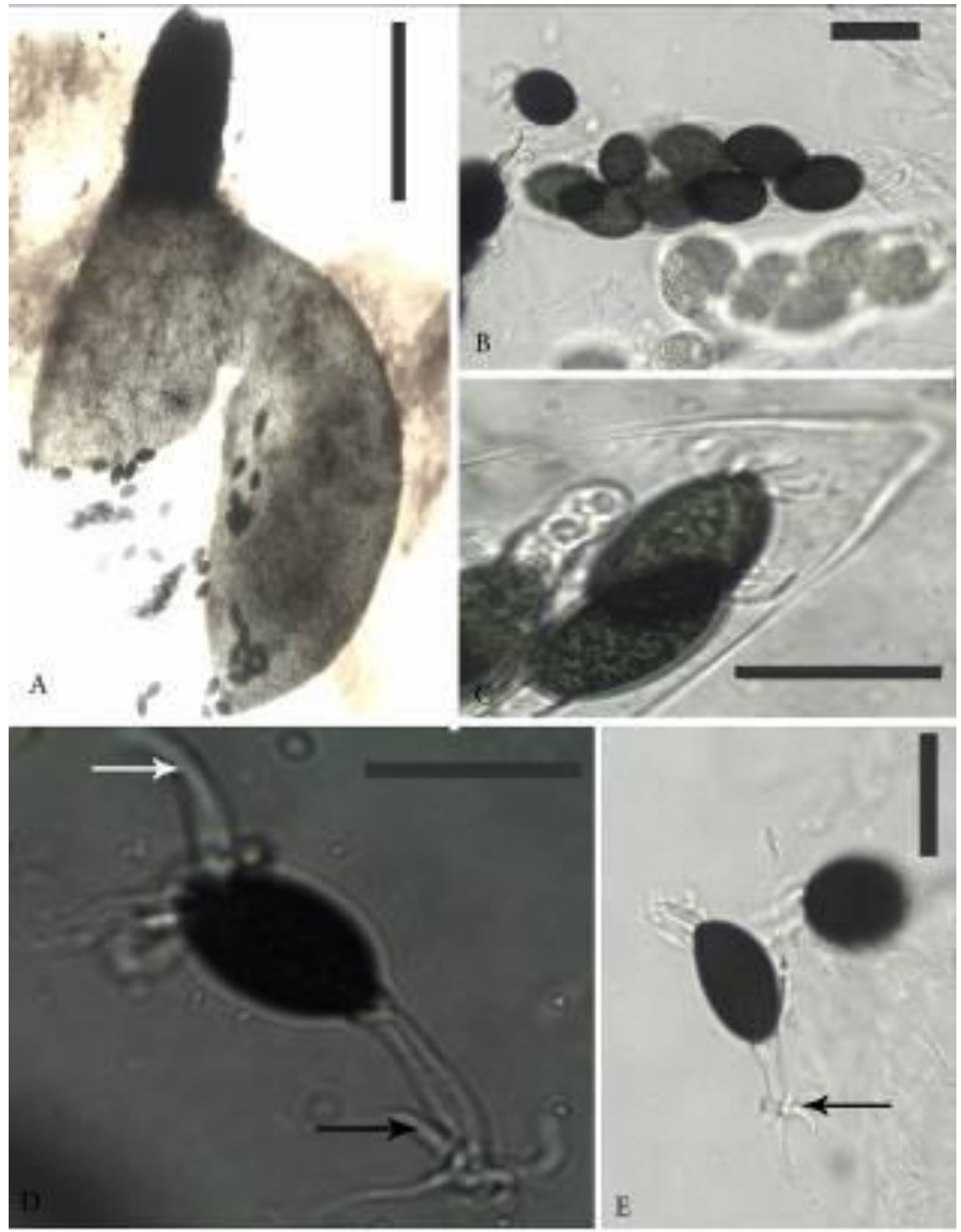

Fig. 5 - Podospora communis (KWSNNP001-2009). A Ascoma squash. B Asci with ascospores in different stages of maturity. C Pointed ascus tip. D Free mature ascospore showing 4 apical (white arrow) and 4 basal caudae (black arrow). E Free mature ascospore showing 4 basal caudae (arrow). Scale bars: $\mathbf{A}=200 \mu \mathrm{m}, \mathbf{B}, \mathbf{E}=50 \mu \mathrm{m}, \mathbf{C}-\mathbf{D}=20 \mu \mathrm{m}$. 


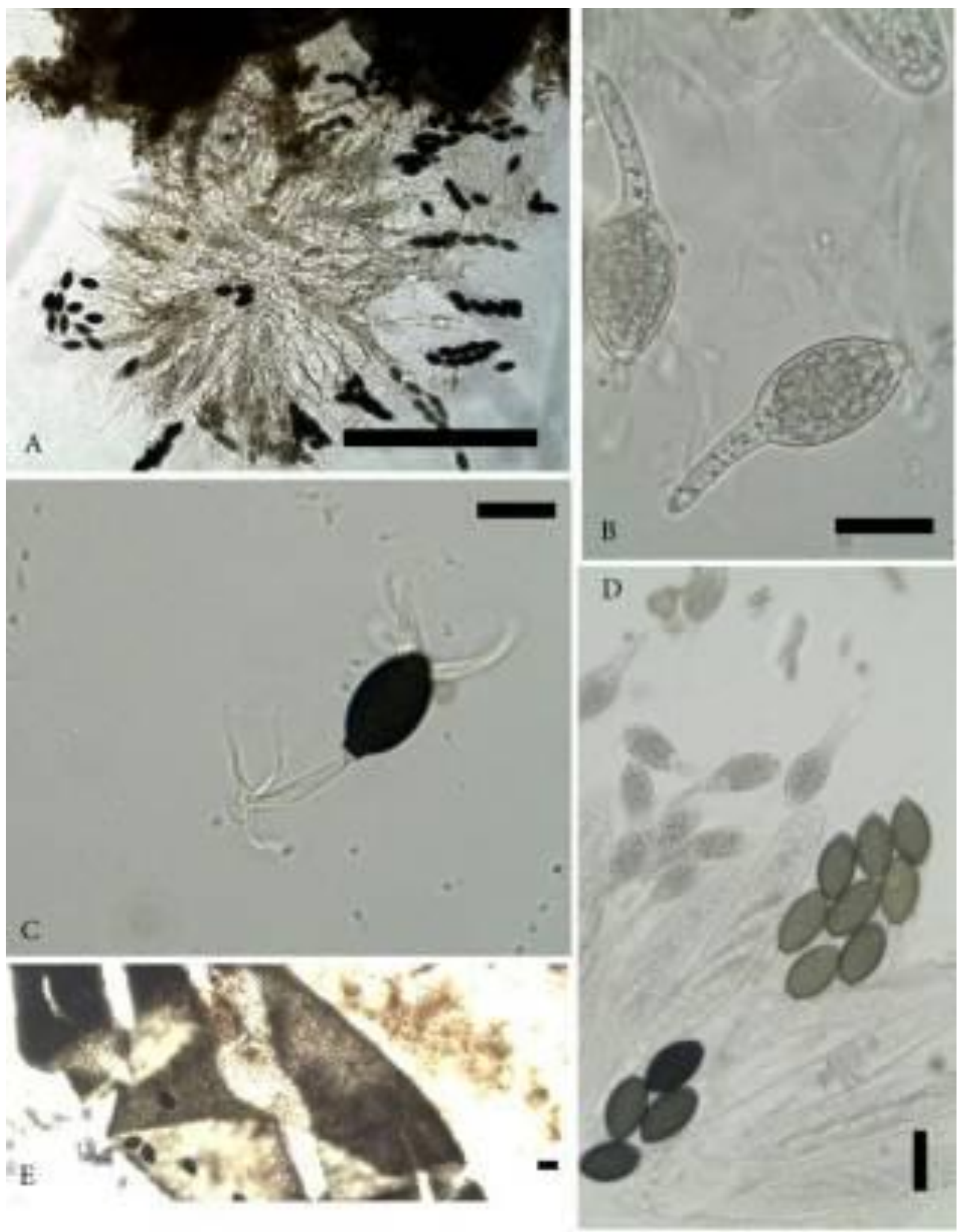

Fig. 6 - Podospora communis (KWSNNP001-2009). A Centrum contents from squashed ascoma. B Immature ascospores. C Mature ascospore. D Ascospores at various stages of maturity. E Details of exoperidium. Scale bars: A, $\mathbf{E}=200 \mu \mathrm{m}, \mathbf{B}=20 \mu \mathrm{m}, \mathbf{C}-\mathbf{D}=50 \mu \mathrm{m}$. 
altitude 1877 m, Cape buffalo, 18 August 2009, P. Mungai, KWSNNP001-2009; Nairobi National Park, UTM37 0252737 M 9847748, altitude $1879 \mathrm{~m}$, impala, 18 August 2009, P. Mungai, KWSNNP003-2009; UTM37 0252737 M 9847748, altitude $1878 \mathrm{~m}$, black rhinoceros, 18 August 2009, P. Mungai, KWSNNP004-2009; GPS UTM37 0253662 M9847748, altitude $1877 \mathrm{~m}$, giraffe, 18 August 2009, P. Mungai KWSNNP005-2009; UTM37 0254976 M 9850544, altitude $1877 \mathrm{~m}$, hippopotamus, 18 August 2009, P. Mungai, KWSNNP006-2009; GPS 37M0255191 9849808, altitude $1693 \mathrm{~m}$, bushed grassland, Cape buffalo, 20 August 2010, P. Mungai, KWSNNP015-2010; Aberdare Country Club Game Sanctuary, Central Province, GPS S00 19 28.1 E03655 54.3, altitude $2061 \mathrm{~m}$, impala, 30 August 2009, P. Mungai, KWSACC001-2009; GPS S00 19'28.1”, E036 55 54.3", altitude $2161 \mathrm{~m}$, bushed grassland, zebra, 30 August, 2009, P. Mungai, KWSACC002-2009; GPS S00 $19 \quad 28.1$ E036 55'54.3", altitude $2161 \mathrm{~m}$, bushed grassland, giraffe, 30 August 2009. P. Mungai, KWSACC003-2009; Aberdare Nairobi National Park, Central Province, GPS S00 19'28.1" E036 55'54.3", altitude $2061 \mathrm{~m}$, black rhinoceros, 29 August 2009, P. Mungai, KWSANP004-2009; GPS S00 21'26.4” E036 51'23.8”, altitude $2004 \mathrm{~m}$, montane forest, giant forest hog, 29 August 2009, P. Mungai, KWSANP001-2009; GPS S00 20'23.2" E036 47'11.1", altitude $2075 \mathrm{~m}$, montane forest, waterbuck, 29 August 2009, P. Mungai, KWSANP005-2009; Tsavo East National Park, Coast Province, GPS S03 02'29.7" E038 41'35.8', altitude $354 \mathrm{~m}$, 27 August 2009, P. Mungai, KWSTE004-2009; GPS S03 02'24.9” E038 42'57.1”, altitude 343 m, waterbuck, 27 August 2009, P. Mungai KWSTE006-2009; GPS S03 21'666" E038 38'772", altitude $514 \mathrm{~m}$, riverine, African elephant, 23 September 2008, P. Mungai, KWSTE007-2008.

Notes - The Kenyan collections have typical features for the species and compare well with descriptions of same species in previous examinations (Lundqvist 1972, Doveri 2004, Bell 2005). Podospora communis belongs to the sect. Malinvernia noted for having clavate or dumb-bell shaped immature ascospores with complex gelatinous equipment at maturity, and peridium lacking a middle gelatinous layer. The main diagnostic characters for $P$. communis include its long, cylindrical pedicel equipped with four short, claw-like, independent gelatinous caudae on the lower end and the presence of four similar caudae on the apex of the spore head (dark cell) (Lundqvist 1972). However, our Kenyan collections have some slight variance with the Australian collection which had three basal caudae (Bell 2005). P. spinulosa R.S. Khan \& Cain, in the same section, is differentiated from $P$. communis by having short spinules on perithecial neck and more caudae at the base of the pedicel; P. hyalopilosa (R. Stratton) Cain has hyaline perithecial neck hairs and a pedicel with only a single apical cauda; $P$. multicaudiculata Cailleux has a slightly hairy neck, shorter asci and multiple but simpler structured caudae; P. austrohemisphaerica N. Lundq. has rigid neck hairs, larger spores, multiple caudae, usually four at each pole, additonal shorter caudae at base of pedicel and a sheath covering the pedicel and spore head; the neck of $P$. deropodalis R.S. Khan \& Cain is covered with short spinules, it has smaller spores but with longer pecidels and variable number of upper caudae and fewer lower caudae; P. alexandri Doveri is similar but can be differentiated by its larger ascospores and the inconspicuous caudae when mounted in water (Doveri 2004a, 2004b, 2008). Podospora communis is the most widespread and commonest species occurring on several wildlife dung types in Kenya (Table 1).

Podospora minor Ellis \& Everh., Amer. Nat. 31: 341, 1897. (Figs. 7A-F, 8A-D, 9A-H)

$\equiv$ Sordaria minor (Ellis \& Everh) Sacc. \& P. Syd., Syll. Fung. 14: 493, 1899.

$\equiv$ Pleurage minor (Ellis \& Everh) Griffiths, Mem. Torrey Bot. Club 11: 67, 1901.

Ascomata perithecioid, superficial, 450-810 $\mu \mathrm{m}$ high., 165-470 $\mu \mathrm{m}$ diam., scattered, black, opaque, coriaceous, with short rigid septate, brown and hyaline tipped hairs $23.5-53 \times 3-4 \mu \mathrm{m}$, conical to pyriform. Neck black, cylindrical, 150-200 × 200-225 $\mu \mathrm{m}$, adorned with stiff septate hyaline tipped hairs. Peridium coriaceous, pseudo-bombardioid, 4layered; outermost peridial wall layer 


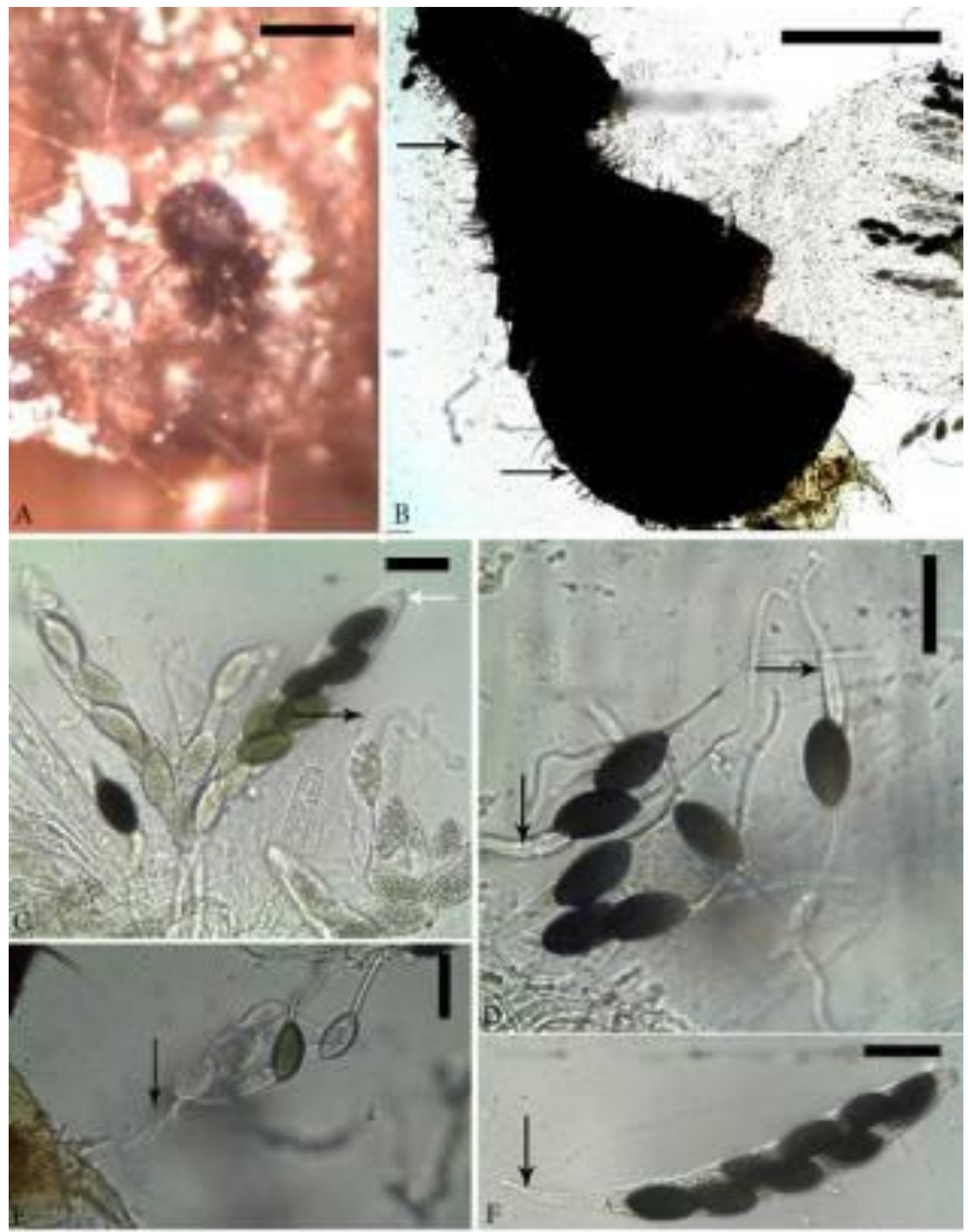

Fig. 7 - Podospora minor (KWSACC002-2009). A Ascoma on dung. B Squashed ascoma mount, note hairs (arrow). C Mature and immature asci and ascospores, note apex (white arrow) and gelatinous equipment (black arrow). D-E Free mature and immature ascospores, note caudae with circinate ends (arrows). F Ascus, showing spore arrangement and stipe (arrow). Scale bars: $\mathbf{A}=$ $500 \mu \mathrm{m}, \mathbf{B}=200 \mu \mathrm{m}, \mathbf{C}-\mathbf{F}=50 \mu \mathrm{m}$. 
Mycosphere Doi 10.5943/mycosphere/3/6/12

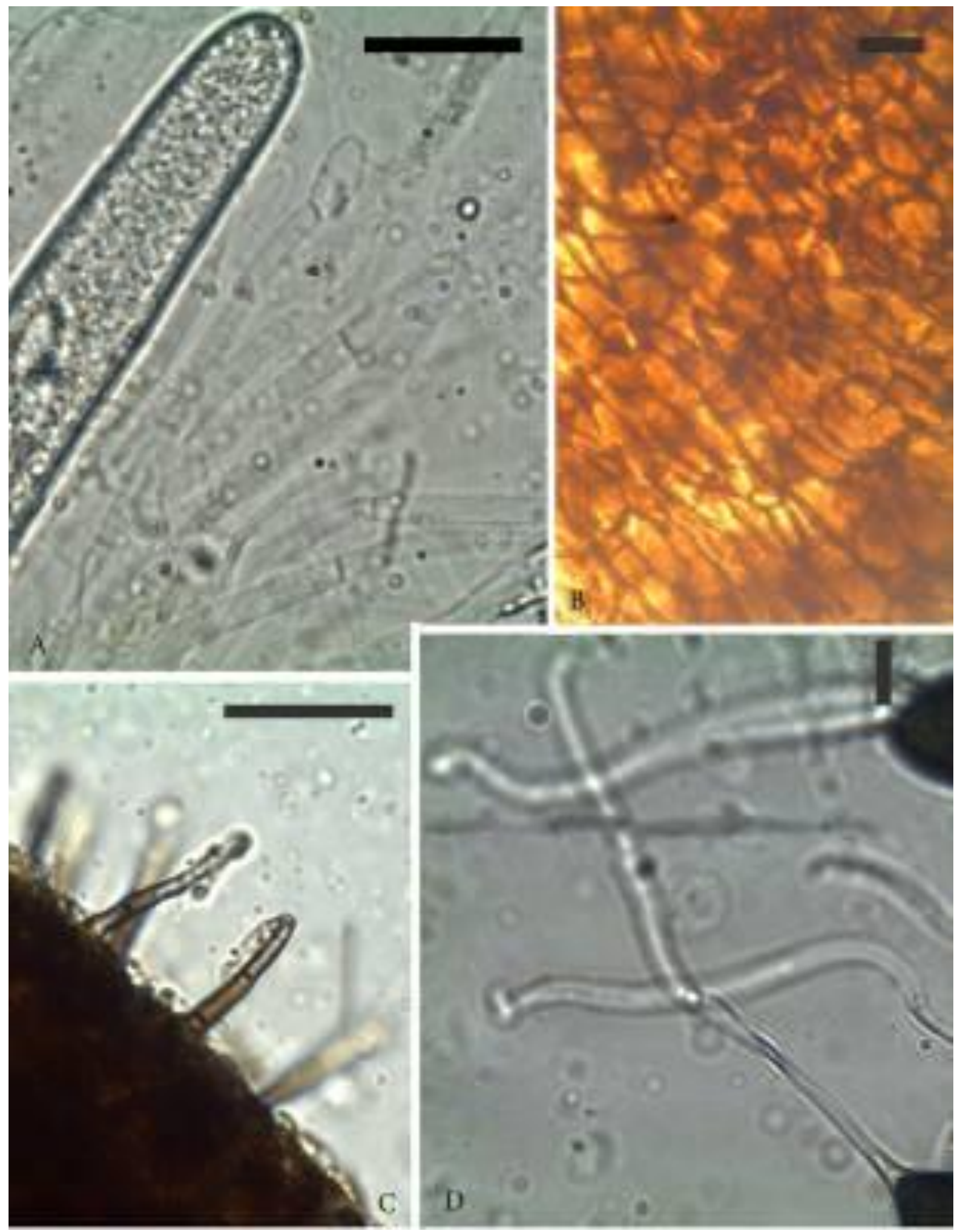

Fig. 8 - Podospora minor (KWSACC002-2009). A Paraphyses. B Details of peridium. C Details of ascomatal hairs. D Details of caudae. Scale bars: $\mathbf{A}-\mathbf{D}=20 \mu \mathrm{m}$. 
Table 1 Occurrence and distribution of Podospora spp. in study area

\begin{tabular}{|c|c|c|c|c|c|c|}
\hline \multirow[t]{2}{*}{ Fungi name } & \multicolumn{2}{|c|}{ Dung type } & \multirow{2}{*}{$\begin{array}{l}\text { Habitat } \\
\text { riverine }\end{array}$} & \multirow{3}{*}{$\begin{array}{c}\begin{array}{c}\text { GPS/Altitude } \\
\text { (m asl) }\end{array} \\
\text { S03 } 21666\end{array}$} & \multicolumn{2}{|l|}{ Date } \\
\hline & KWSTE005A-2009 & African elephant & & & E038 38.772 & 514 \\
\hline Podospora anserina & & & & & & \\
\hline Podospora anserina & KWSANP004-2009 & black rhinoceros & montane forest & $\mathrm{S} 00^{\circ} 21420$ & $\mathrm{E} 036^{\circ} 5255.9$ & 2076 \\
\hline Podospora anserina & KWSNNP004-2009 & Cape buffalo & bushed grassland & 0252737 & 9847748 & 1876 \\
\hline Podospora anserina & KWSTE007A-2008 & zebra & riverine habitat & S03 21064 & E038 37501 & 514 \\
\hline Podospora anserina & KWSTE005B-2009 & dikdik & riverine & S0302 297 & $\mathrm{E} 038^{\circ} 41358$ & 354 \\
\hline Podospora anserina & KWSACC003-2009 & giraffe & bushed grassland & S00 19281 & $\mathrm{E} 036^{\circ} 55543$ & 2161 \\
\hline Podospora anserina & KWSNNP012-2009 & hippopotamus & bushed grassland & S01 20501 & $\mathrm{E} 036^{\circ} 47513$ & 1695 \\
\hline Podospora argentinensis & KWSNNP016-2010 & zebra & wooded grassland & 37M0257082 & 9850692 & 1668 \\
\hline Podospora argentinensis & KWSACC002-2009 & zebra & bushed grassland & $\mathrm{S} 00^{\circ} 19281$ & $\mathrm{E} 036^{\circ} 5554.3$ & 2161 \\
\hline Podospora australis & KWSACC002-2009 & zebra & bushed grassland & S00 19281 & $\mathrm{E} 036^{\circ} 5554.3$ & 2161 \\
\hline Podospora australis & KWSNNP016-2010 & zebra & wooded grassland & 37M0255297 & 9848528 & 1693 \\
\hline Podospora australis & $\begin{array}{l}\text { KWSNNP017B- } \\
2010\end{array}$ & giraffe & wooded grassland & 37M0257532 & 9848948 & 1647 \\
\hline Podospora australis & $\begin{array}{l}\text { KWSNNP017A- } \\
2010\end{array}$ & giraffe & wooded grassland & 37M0257532 & 9848948 & 1647 \\
\hline Podospora communis & KWSTE007-2008 & African elephant & riverine & S03 21666 & E038 38.772 & 514 \\
\hline Podospora communis & KWSNNP004-2009 & black rhinoceros & bushed grassland & $\begin{array}{l}\text { UTM370252 } \\
737\end{array}$ & M9847748 & 1878 \\
\hline Podospora communis & KWSANP004-2009 & black rhinoceros & montane forest & S00 19281 & E036 55543 & 2061 \\
\hline Podospora communis & KWSNNP001-2009 & Cape buffalo & Grassland & $\begin{array}{l}\text { UTM370253 } \\
7515\end{array}$ & M9849130 & 1877 \\
\hline Podospora communis & KWSNNP015-2010 & Cape buffalo & bushed grassland & 37M0255191 & 9849808 & 1693 \\
\hline Podospora communis & KWSACC002-2009 & zebra & bushed grassland & SO0 19281 & E036 55543 & 2161 \\
\hline Podospora communis & KWSTE004-2009 & zebra & Savannah & S0302 297 & E03841358 & 354 \\
\hline Podospora communis & KWSANP001-2009 & giant forest hog & montane forest & $\mathrm{S} 00^{\circ} 21264$ & E036 51238 & 2004 \\
\hline Podospora communis & KWSACC003-2009 & giraffe & bushed grassland & S00 19281 & E036 55543 & 2161 \\
\hline Podospora communis & KWSNNP006-2009 & hippopotamus & Wetland & $\begin{array}{l}\text { UTM370254 } \\
976\end{array}$ & M9850544 & 1877 \\
\hline Podospora communis & KWSNNP003-2009 & impala & Grassland & $\begin{array}{l}\text { UTM370252 } \\
737\end{array}$ & M9847748 & 1879 \\
\hline Podospora communis & KWSACC001-2009 & impala & bushed grassland & S00 19281 & E036 55543 & 2061 \\
\hline Podospora communis & KWSANP005-2009 & waterbuck & montane forest & $\mathrm{S} 00^{\circ} 20232$ & E036 47111 & 2075 \\
\hline Podospora communis & KWSTE006-2009 & waterbuck & Riverine & S0302 249 & E0384257 1 & 343 \\
\hline Podospora minor & KWSACC002-2009 & zebra & bushed grassland & S00 19281 & E036 55543 & 2161 \\
\hline
\end{tabular}


composed of textura angularis thick-walled brown elongated polygonal cells; second layer from outside made up of hyaline, thick-walled gelatinous polygonal cells; third layer of darker, thick-walled flattened cells; fourth layer composed of lighter thin-walled polygonal cells. Paraphyses exceeding the asci, filiform above and ventricose below, septate, 3-4.5 $\mu \mathrm{m}$ broad, hyaline. Asci 8-spored, 245-345 × 27.5$43 \mu \mathrm{m}$, cylindrical-clavate, unitunicate, narrow, evanescent, round apex, short stipitate. Ascospores uniseriate to biseriate, at first hyaline, single-celled, fusiform then cylindrical; spore head $37.5-47.5 \times 20.5-25 \mu \mathrm{m}$ at maturity, slightly inequilateral, black-brown, ellipsoidal to fusiform; germ pore apical; pedicel hyaline, cylindrical or clavate, broader at the tip; upper cauda longer and broader, 75.5-126.5 × 9-12 $\mu \mathrm{m}$, lash-like, appearing striated and furrowed, eccentric, not covering the germ pore, hollow; lower cauda shorter and narrower, 46-73.5 $\times$ 4.5-5 $\mu \mathrm{m}$, hyaline, hollow, attached to distal end of the pedicel, tips circinate.

Material examined - one specimen on dung incubated for 42 days - KENYA, Aberdare Country Club Game Sanctuary, Central Province, GPS S00 19'28.1" E036 55'54.3", altitude $2161 \mathrm{~m}$, zebra, 30 August 2009, P. Mungai, KWSACC002-2009.

Notes - Podospora minor is in the section Podospora. The ascospores of $P$. minor are morphologically similar to those of $P$. fimiseda (Ces. \& De Not.) Niessl. belonging to the same section but are slightly smaller. The characters of $P$. minor seem to be intermediate between $P$. appendiculata (Auswer.) Niessl and $P$. fimiseda. This Kenyan collection has broader asci and larger ascospores in comparison to the specimen examined by Mirza \& Cain (1969). This species appears to be rare in wildlife dung and we make the first record of $P$. minor in Kenya.

\section{Ecology}

Podospora communis (50\%), $P$. anserina $(25 \%)$ and $P$. australis $(14.3 \%)$ are the most common on the dung types examined. (Adopted from Zak \& Willig 2004)

Commonly collected (African elephant, black rhinoceros, zebra, Cape buffalo and giraffe) and rarely collected dung types (dikdik, hippopotamus, giant forest hog, impala and waterbuck) were found in different habitat types, incubated and examined (Table 1).

Type, structure, texture, moisture contents and age of dung collected are important variables for coprophilous Podospora. The structure and composition of dung varies according to the animal species voiding it. The dung of African elephant is usually voided as one or two very coarse large randomly dispersed piles; black rhinoceros and hippopotamus dung are also large, less coarse and look very similar. However, black rhinoceros dung was always voided at one point.

Hippopotamus is territorial and thus usually marks its territory by splashing dung on vegetation within the territory and therefore its dung is always a mixture of several days voided biomass. The texture of Cape buffalo dung was the finest for the large mammals sampled. The rest of the wild animals sampled void dung in form of pellets of various shapes and sizes. Zebra has the largest pellets followed by giraffe while dikdik has the smallest dung pellets sampled.

Moisture content of the dung has a great influence on Podospora sporulation. Extremely wet dung samples such as those from the very rainy mountainous Aberdares National Park needs thorough air-drying after sampling. This was noted to have some influence on Podospora species occurrence.

The time the dung is exposed since voiding and the time it is sampled is also a crucial variable. Very old and dry dung does not yield as many isolates as freshly voided dung.

Dung sampled from grassland habitats produced the most Podospora isolates in this study (Table 1).

\section{Conclusion}

Coprophilous Podospora species diversity in Kenya is high and seems to be influenced by a number of factors both biotic and abiotic. The correlation observed between the structure of dung and type on one hand and Podospora on the other hand need further elucidation. This study has helped create awareness on the need to understand dung fungi and its ecological roles. It is hoped that there will be a shift in policy to embrace fauna, 

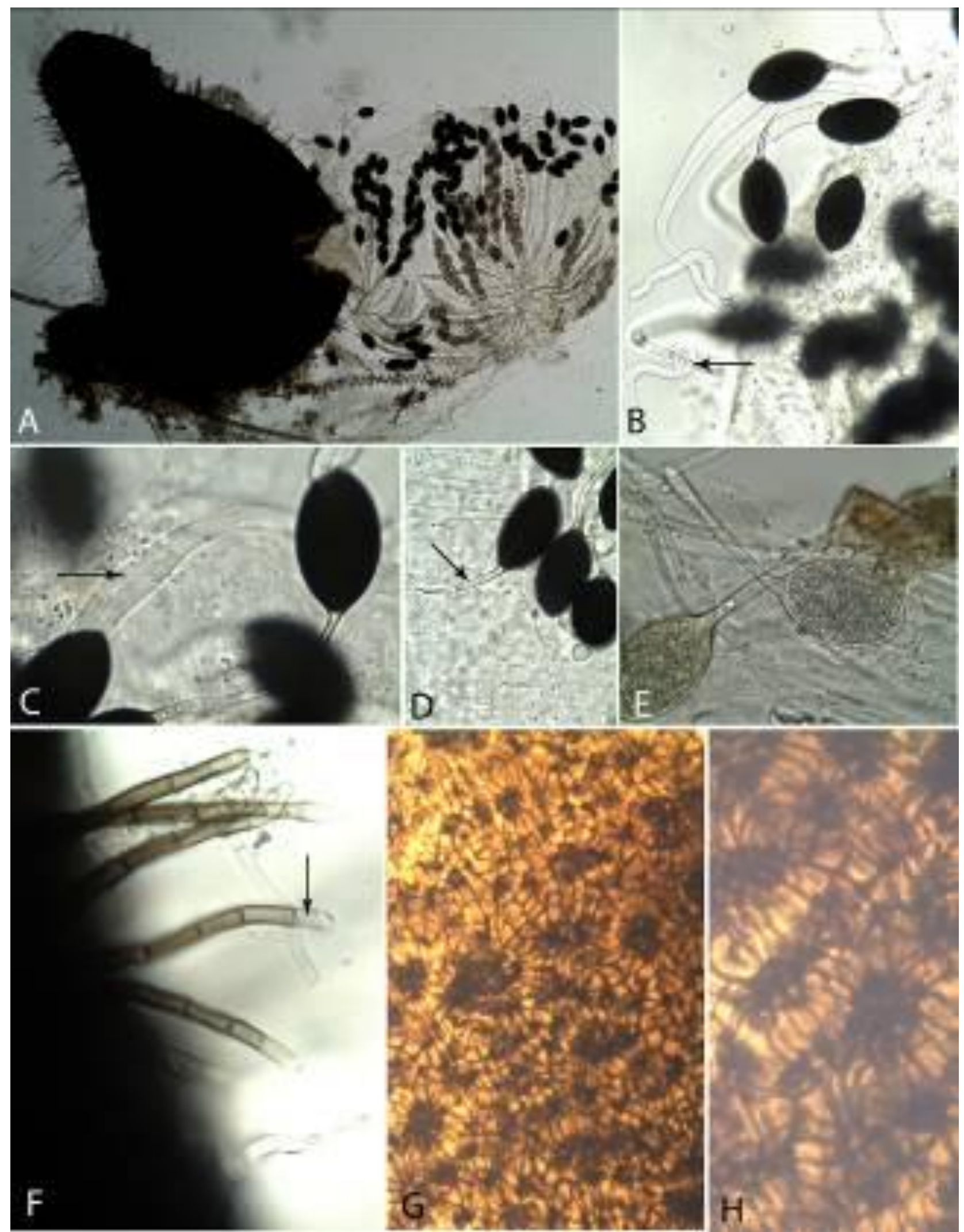

Fig. 9 - Podospora minor (KWSACC002-2009). A Squashed ascoma. B Ascospores showing caudae, note coiled ends (arrow). C Details of cauda, note the furrows (arrow). D Details of pedicel, note the curvature (arrow). E Immature ascospores. F Ascomatal hairs, note the hyaline tips. G-H Ascomatal wall. Scale bars: $\mathbf{A}=200 \mu \mathrm{m}, \mathbf{B}, \mathbf{G}=50 \mu \mathrm{m}, \mathbf{C}-\mathbf{F}, \mathbf{H}=20 \mu \mathrm{m}$. 
flora and fungi (3Fs) as a new paradigm fo Kenyan biodiversity conservation and management.

\section{Acknowledgements}

This work was funded by Kenya Wildlife Service (KWS), Mushroom Research Foundation and Novozymes A/S of Denmark. We are grateful to the Director (KWS) and Deputy Director, Biodiversity Research and Monitoring Division, for allowing us to conduct the study within the National Parks and Reserves in Kenya. We wish to appreciate and thank Dr Francesco Doveri for taking a lot of his time to do a thorough taxonomic correction of this paper. It is kindly noted that without his thoughtful advice and input this work could not have been possible. We also feel highly indebted to staff and students of the Institute of Excellence in Fungal Research, School of Science, Mae Fah Luang University, Thailand and KWS colleagues, Mr Patrick Omondi, Dr Samuel Andanje, Dr Francis Gakuya, Mr Moses Otiende and Mrs Elsie Wambui Maina for their support during this study. Ms Asenath Akinyi assisted in laboratory examination, grammar and spelling checks of the text and neatly arranging the tabulated results.

\section{References}

Abdullah SK, Rattan SS. 1978 - Zygopleurage, Tripterosporella and Podospora (Sordariaceae: Pyrenomycetes) in Iraq. Mycotaxon 7, 102-116.

Bell A. 1983 - Dung Fungi. An Illustrated Guide to Coprophilous Fungi in New Zealand. Victoria University Press, Wellington. $88 \mathrm{pp}$.

Bell A. 2005 - An illustrated guide to the coprophilous ascomycetes of Australia. CBS Biodiversity Series 3. Centraalbureau voor Schimmelcultures, Utrecht, The Netherlands. 172 pp.

Bell AE, Mahoney DP. 1995 - Coprophilous fungi in New Zealand. I. Podospora species with swollen agglutinated perithecial hairs. Mycologia 87, 375396.
Bell AE, Mahoney DP. 1997 - Coprophilous fungi in New Zealand. II. Podospora species with coriaceous perithecia. Mycologia 89, 908-915.

Cain RF. 1934 - Studies of coprophilous Sphaeriales in Ontario. University of Toronto Studies in Biology Series 38, $1-126$.

Chang J-H Wang Y-Z. 2005 - A new species of Podospora from Taiwan. Botanical Bulletin of Academia Sinica 46, 169173.

Doveri F. 2004a - Fungi Fimicoli Italici. Associazione Micologica Bresadola, Trento, Italy.

Doveri F. 2004b - Podospora alexandri, una Nuova Specie Fimicola dall'Italia. Rivista di Micologia 47(3), 211-221.

Doveri F. 2008 - Pagine di Micologia, Associazone Micologica Bresadola Centro Studi Micologici.

Kirk PM, Cannon PF, David JC, Stalpers JA. 2008 - (eds) Ainsworth \& Bisby's dictionary of the Fungi, 10th Edition. CABI, Wallingford.

Krug JC, and Khan RS. 1989 - New records and new species of Podospora from east Africa. Canadian Journal of Botany 7, 1174-1182.

Lundqvist N. 1969 - Zygopleurage and Zygospermella (Sordariaceae s. lat., Pyrenomycetes). Botaniska Notiser 122, 353-374.

Lundqvist N. 1972 - Nordic Sordariaceae s.lat. Symbolae Botanicae Upsaliensis 20, 1374.

Lundqvist N. 1973 - Studia Fungorum Fimi.1. New Records of Podosporae and a new species, $P$. papilionacea. Svensk Botanisk Tidskrift 67, 34-52.

Mirza JH, Cain RF. 1969 - Revision of the genus Podospora. Canadian Journal of Botany 49, 1999-2048.

Mungai P, Hyde KD, Cai L, Njogu J, Chukeatirote E. 2011 - Coprophilous ascomycetes of northern Thailand. Current Research in Environmental and Applied Mycology 1, 135-159.

Mungai PG, Njogu JG, Chukeatirote E, Hyde KD. 2012a - Studies of coprophilous 
ascomycetes in Kenya. Ascobolus species from wildlife dung. Current Research in Applied and Environmental Mycology 2, 1-16.

Mungai PG, Njogu JG, Chukeatirote E, Hyde KD. 2012b - Coprophilous ascomycetes in Kenya. Saccobolus species from wildlife dung. Mycosphere 3(2), 111-129.

Mungai PG, Chukeatirote E, Njogu JG, Hyde KD. 20102c - Studies of coprophilous ascomycetes in Kenya: Sordariales from wildlife dung. Mycosphere 3(4), 437-448.

Mungai PG, Njogu JG, Chukeatirote E, Hyde
KD. 2012d - Studies of coprophilous ascomycetes in Kenya - Schizothecium from wildlife dung. Current Research in Applied and Environmental Mycology 2(1), 84-97.

Richardson MJ. 2001 - Coprophilous fungi from Brazil. Brazilian Archives of Biology and Technology 44, 283-289.

Zak JC, Willig MR. 2004 - Fungal biodiversity patterns. In: Biodiversity of Fungi, Inventory and Monitoring Methods (eds. GM Muller, FG Bills, MS Foster). Elsevier Academic Press, San Diego, California pp 59-75. 